\title{
Partly noiseless encoding of quantum information in quantum dot arrays against phonon-induced pure dephasing
}

\author{
A. Grodecke* and P. Machnikowski \\ Institute of Physics, Wroctaw University of Technology, 50-370 Wroctaw, Poland
}

\begin{abstract}
We show that pure dephasing of a quantum dot charge (excitonic) qubit may be reduced for sufficiently slow gating by collectively encoding quantum information in an array of quantum dots. We study the role of the size and structure of the array and of the exciton lifetime for the resulting total error of a single-qubit operation.
\end{abstract}

\section{INTRODUCTION}

The idea of implementing quantum information processing schemes on charge states in quantum dot (QD) systems 1.2 has recently been supported by an experimental demonstration ${ }^{3}$. On the other hand, it has been proposed that quantum bits encoded in electron spins (which have much longer dephasing times) may be optically controlled by inducing charge dynamics within various schemes exploiting selection rules and Pauli blocking 4,5,6.7. Moreover, the demonstration of a QD-based photodiode operating in the quantum coherent regime ${ }^{8}$ shows great promise for quantum optoelectronic applications. These experimental achievements and theoretical proposals have driven a lot of investigation on coherence properties of QD systems, in particular under external driving of carrier dynamics.

Limitations to the coherent control of confined carriers in QDs result, on one side, from finite lifetime of charge states used for information encoding; one restriction is imposed, for instance, by radiative decay (on nanosecond time scale ${ }^{9}$ ) of confined excitons that seemed to be the most natural charge qubit systems due to relative ease of ultrafast optical driving ${ }^{2}$. On the other side, optical experiments ${ }^{10}$ have demonstrated that pure dephasing processes destroy coherence of the system state to a large extent within a few picoseconds after creating the state. This has been attributed to carrier-phonon interaction ${ }^{11.12}$ and interpreted in terms of spontaneous relaxation of the lattice after an optically induced change of the confined charge distribution, accompanied by emission of phonon wave packets ${ }^{13.14}$, which creates a kind of which way trace in the crystal ${ }^{15}$ and results in dephasing. The same phonon response to the charge evolution leads also to dephasing in optical spin control schemes ${ }^{5,16}$.

The phonon-induced dephasing effect may be avoided by driving the system evolution adiabatically with respect to phonon dynamics ${ }^{5,17}$, which requires long driving times and leads to growing probability of exciton decay during the operation. Optimal driving conditions correspond to a tradeoff between these two dephasing effects $^{17}$. As a result, maximum fidelity of a coherent quantum control operation on a given system is limited.

One way to partly circumvent this limitation is optimizing the pulse shape ${ }^{18.19}$. Another solution might be to modify the properties of the phonon reservoir by placing the dot near the crystal surface ${ }^{20}$ or in an acoustic cavity ${ }^{21}$. An alternative approach is to overcome the physical restrictions imposed by dephasing processes on the quantum-logical level. If the reservoir acts collectively on a register composed of a number of charge qubits then subspaces exist which are safe against decoherence $22,23,24,25$. In principle, this idea can be used to implement robust logical qubits on QD arrays ${ }^{1.26}$. In practice, however, protection against decoherence based on collective interaction with the environment (closely related to the subradiance effect) may be difficult to achieve in these artificial systems since it requires that the transition energies in all QDs are the same. Moreover, the phonon-induced pure dephasing effect involves a range of modes selected by the system geometry in such a way that reservoir correlation length roughly corresponds to the single QD size which apparently precludes any collective interaction with a number of QDs.

In this paper we show that, in spite of these difficulties, partly noiseless encoding against the pure dephasing effect is possible. We focus our discussion on the case of an excitonic charge qubit. In analogy to the original idea of noiseless encoding ${ }^{22}$, we will identify two states of an array of quantum dots which span a subspace (logical qubit) that, under appropriate driving conditions, allows performing single-qubit operations at reduced error, i.e., with relatively little lattice response, compared to switching of a single excitonic physical qubit. Physically, the collective encoding approach in this case relies on the fact that the pure dephasing effect is of dynamical character and results exclusively from nonadiabaticity of the driving 17 . For a given speed of rotation in the qubit space, it involves only slow modes that cannot follow the evolution of charge distribution, while faster modes follow adiabatically and reversibly. In this way, only a long wave length part of all the coupled modes is relevant for the dynamics. Since the range of these slow modes is selected dynamically, independently of the system geometry, it may correspond (under suitable driving conditions) to wave lengths larger than the register (QD array) size, thus allowing for collective interaction.

In the present proposal, the logical qubit is defined on a system of $N=2^{n}$ quantum dots (physical qubits) in such a way that the logical $|1\rangle$ state corresponds to the presence of an exciton in each of the appropriately chosen $N / 2$ dots, while in the logical $|0\rangle$ state the other half of the dots are occupied. With the correct definition of the logical qubit, the excitations of the phonon reservoir 
induced by the evolution of charges in all QDs interfere destructively in the long wave length sector, leading to a decrease of dephasing. The proposed scheme works only for relatively slow evolution and the resulting fidelity gain is again restricted by finite lifetimes. We will study how much the overall error may be reduced depending on the exciton lifetime and the strength of other decoherence processes. It should be stressed that the scheme proposed here does not assume that the individual dots in the register are identical.

The paper is organized as follows. In Sec. I we introduce the model of the QD array implementing the logical qubit. Next, in Sec. III] we present the general method for describing the effects of phonon perturbation on an arbitrary operation on a logical qubit. Sec. IV contains the results, discussing the error reduction for various layouts of the logical qubit. Sec. $\mathbb{\nabla}$ concludes the paper with final remarks.

\section{MODEL}

As a model of the physical QD array implementing the logical qubit, we consider $N$ quantum dots, each approximated by a two-level system corresponding to the absence or presence of an exciton with a fixed polarization, with transition energies $\epsilon_{l}, l=1, \ldots N$. The wave functions of carriers confined in different dots do not overlap so that no phonon-assisted transfer is possible. We will be interested in driving the system with long, i.e., spectrally narrow pulses so that excitations of optical phonons may be excluded. If the electron and hole wave functions in each dot overlap the piezoelectric coupling is very weak due to charge cancellation ${ }^{11}$. Therefore, we assume that the confined carriers interact only with longitudinal acoustic phonons via deformation potential coupling. The Hamiltonian of this semiconductor system is then

$$
\begin{aligned}
H_{\mathrm{sc}} & =\sum_{n} \epsilon_{n} a_{n}^{\dagger} a_{n}+\sum_{\boldsymbol{k}} \hbar \omega_{\boldsymbol{k}} b_{\boldsymbol{k}}^{\dagger} b_{\boldsymbol{k}} \\
& +\sum_{n, \boldsymbol{k}} a_{n}^{\dagger} a_{n} f_{n}(\boldsymbol{k})\left(b_{\boldsymbol{k}}^{\dagger}+b_{-\boldsymbol{k}}\right)
\end{aligned}
$$

where $a_{n}^{\dagger}, a_{n}$ are fermionic creation and annihilation operators for the exciton in the $n$th dot, $b_{\boldsymbol{k}}^{\dagger}, b_{\boldsymbol{k}}$ are bosonic operators for phonon modes, $\omega_{\boldsymbol{k}}=c k$ are the corresponding frequencies ( $c$ is the speed of sound), and $f_{n}(\boldsymbol{k})$ are the carrier-phonon coupling constants for an exciton in the $n$th dot with the symmetry $f_{n}(\boldsymbol{k})=f_{n}^{*}(-\boldsymbol{k})$. Assuming, for simplicity, that the exciton state in the $n$th dot is described by a product of electron and hole wave functions $\psi_{n}^{(\mathrm{e} / \mathrm{h})}\left(\boldsymbol{r}-\boldsymbol{r}_{n}\right)$, where $\boldsymbol{r}_{n}$ is the position of the dot, the coupling constants may be written as 27

$$
f_{n}(\boldsymbol{k})=\sqrt{\frac{\hbar k}{2 \rho_{\mathrm{c}} v c}}\left(\sigma_{\mathrm{e}}-\sigma_{\mathrm{h}}\right) e^{-i \boldsymbol{k} \cdot \boldsymbol{r}_{n}} \mathcal{F}_{n}(\boldsymbol{k}),
$$

with the formfactors

$$
\begin{aligned}
\mathcal{F}_{n}(\boldsymbol{k})= & \frac{1}{\sigma_{\mathrm{e}}-\sigma_{\mathrm{h}}} \int d^{3} r d^{3} r^{\prime}\left|\psi_{n}^{(\mathrm{e})}(\boldsymbol{r})\right|^{2}\left|\psi_{n}^{(\mathrm{h})}\left(\boldsymbol{r}^{\prime}\right)\right|^{2} \\
& \times\left(\sigma_{\mathrm{e}} e^{-i \boldsymbol{k} \cdot \boldsymbol{r}}-\sigma_{\mathrm{h}} e^{-i \boldsymbol{k} \cdot \boldsymbol{r}^{\prime}}\right) .
\end{aligned}
$$

Here $\rho_{\mathrm{c}}$ denotes the crystal density, $v$ is the normalization volume of the phonon modes, and $\sigma_{\mathrm{e} / \mathrm{h}}$ are the deformation potential constants for electrons and holes.

The Hamiltonian $H_{\mathrm{sc}}$ [Eq. (10] has the structure of the independent boson model and may be diagonalized exactly $^{28}$. To this end, one defines the operator

$$
\mathbb{W}=\exp \left[\sum_{n, \boldsymbol{k}} a_{n}^{\dagger} a_{n} g_{n}^{*}(\boldsymbol{k}) b_{\boldsymbol{k}}-\text { H.c. }\right],
$$

where $g_{n}(\boldsymbol{k})=f_{n}(\boldsymbol{k}) /\left(\hbar \omega_{\boldsymbol{k}}\right)$, and writes the Hamiltonian $H_{0}$ in terms of the operators

$$
\alpha_{n}=\mathbb{W}^{\dagger} a_{n} \mathbb{W}=a_{n} W_{n}
$$

and

$$
\beta_{\boldsymbol{k}}=\mathbb{W}^{\dagger} b_{\boldsymbol{k}} \mathbb{W}=b_{\boldsymbol{k}}+\sum_{n} \alpha_{n}^{\dagger} \alpha_{n} g_{n}(\boldsymbol{k})
$$

where

$$
W_{n}=\exp \left[\sum_{\boldsymbol{k}} g_{n}^{*}(\boldsymbol{k}) b_{\boldsymbol{k}}-\text { H.c. }\right] .
$$

In the calculations one uses the fact that $\left[W_{n}, W_{m}\right]=0$ for non-overlapping exciton states. The result is

$$
H_{\mathrm{sc}}=\sum_{n} E_{n} \alpha_{n}^{\dagger} \alpha_{n}+\sum_{\boldsymbol{k}} \hbar \omega_{\boldsymbol{k}} \beta_{\boldsymbol{k}}^{\dagger} \beta_{\boldsymbol{k}}
$$

where $E_{n}=\epsilon_{n}-\sum_{\boldsymbol{k}}\left|f_{n}(\boldsymbol{k})\right|^{2} /\left(\hbar \omega_{\boldsymbol{k}}\right)$. The new operators (4) and (5) represent excitons in the dots surrounded by coherent lattice displacement field and phonon modes shifted by the presence of a charge distribution.

We will associate the value $|0\rangle$ of the logical qubit with the state of the QD array in which a certain subset of $N / 2$ dots, labelled by indices $n_{1}, \ldots n_{N / 2}$, is occupied by excitons and the value $|1\rangle$ with the state in which the other $N / 2\left(n_{1}^{\prime}, \ldots n_{N / 2}^{\prime}\right.$, with $n_{l} \neq n_{l^{\prime}}^{\prime}$ for all $\left.l, l^{\prime}=1, \ldots, N / 2\right)$ dots are occupied. Thus, the basis states of the logical qubit are

$$
|0\rangle=a_{n_{1}}^{\dagger} \ldots a_{n_{N / 2}}^{\dagger}|\mathrm{g}\rangle, \quad|1\rangle=a_{n_{1}^{\prime}}^{\dagger} \ldots a_{n_{N / 2}^{\prime}}^{\dagger}|\mathrm{g}\rangle,
$$

where $|g\rangle$ is the ground state of the system (with all dots empty). In our discussion we will assume that a control field is available that couples the relevant pair of states of the multi-QD register and induces a transition without leaving the subspace spanned by these states. In particular, we assume the availability of a control Hamiltonian of the form

$$
H_{\mathrm{C}}=\frac{1}{2} f(t) e^{-i \Delta E t / \hbar} a_{n_{1}}^{\dagger} \ldots a_{n_{N / 2}}^{\dagger} a_{n_{1}^{\prime}} \ldots a_{n_{N / 2}^{\prime}}+\text { H.c. }
$$


which transfers $N / 2$ excitons from one subset of dots to the other. Here $f(t)$ is the envelope of the control field and

$$
\Delta E=\sum_{l=1}^{N / 2} E_{n_{l}}-\sum_{l=1}^{N / 2} E_{n_{l}^{\prime}}
$$

is the difference between the total energies of the logical states $|0\rangle$ and $|1\rangle$. A practical implementation of such a coupling is a separate issue. For transferring a single exciton between two dots, a realization of an effective Hamiltonian of this kind has been proposed, using the Förster coupling and the optical Stark effect ${ }^{29.30}$.

In terms of the redefined degrees of freedom [Eqs. (4) and (5)], the control Hamiltonian (7) to the leading order in phonon coupling reads

$$
\begin{aligned}
H_{\mathrm{C}}= & \frac{1}{2} f(t) e^{-i \Delta E t / \hbar} \alpha_{n_{1}}^{\dagger} \ldots \alpha_{n_{N / 2}}^{\dagger} \alpha_{n_{1}^{\prime}} \ldots \alpha_{n_{N / 2}^{\prime}} \\
& \times\left[1+\sum_{\boldsymbol{k}} G(\boldsymbol{k})\left(\beta_{-\boldsymbol{k}}-\beta_{\boldsymbol{k}}^{\dagger}\right)\right]+\text { H.c. }
\end{aligned}
$$

with

$$
G(\boldsymbol{k})=\left[g_{n_{1}^{\prime}}(\boldsymbol{k})+\ldots+g_{n_{N / 2}^{\prime}}(\boldsymbol{k})\right]-\left[g_{n_{1}}(\boldsymbol{k})+\ldots+g_{n_{N / 2}}(\boldsymbol{k})\right]
$$

The phonon perturbation in Eq. (8) leads to reservoir excitations accompanying any operation on the carrier subsystem but does not drive this subsystem outside the subspace spanned by the logical qubit states.

In our calculations the wave functions of confined electrons and holes will be modelled by identical Gaussians,

$$
\psi(\boldsymbol{r})=\frac{1}{\pi^{3 / 4} l_{z} l} \exp \left(-\frac{x^{2}+y^{2}}{2 l^{2}}\right) \exp \left(-\frac{z^{2}}{2 l_{z}^{2}}\right),
$$

where the parameters $l, l_{z}$ are related to the QD size in the $x y$ plane and along the $z$ direction, respectively. For simplicity, we assume here that the wave functions in all QDs are identical. As shown in the Appendix, including a small variation of dot sizes and relative electron and hole confinement widths will only lead to inessential quantitative corrections. For this choice of wave functions, the formfactor [Eq. (3)] has the explicit form

$$
\mathcal{F}(\boldsymbol{k})=e^{-\left(k_{\perp} l / 2\right)^{2}-\left(k_{z} l_{z} / 2\right)^{2}},
$$

where $k_{\perp}=\left(k_{x}^{2}+k_{y}^{2}\right)^{1 / 2}$.

We will assume that the operation is performed by a Gaussian control pulse,

$$
f(t)=\frac{\hbar \alpha}{\sqrt{2 \pi} \tau_{\mathrm{p}}} e^{-\frac{1}{2}\left(\frac{t}{\tau_{\mathrm{p}}}\right)^{2}}
$$

where $\alpha$ is the angle determining the gate (corresponding to the angle of rotation on the Bloch sphere) and $\tau_{\mathrm{p}}$ is the gate duration.

In Tab. Ithe material parameters (corresponding to an InAs/GaAs system) are collected.

\begin{tabular}{lll}
\hline Deformation potential coupling & $\sigma_{\mathrm{e}}-\sigma_{\mathrm{h}} 8 \mathrm{eV}$ \\
Crystal density & $\rho_{\mathrm{c}}$ & $5360 \mathrm{~kg} / \mathrm{m}^{3}$ \\
Speed of sound (longitudinal) & $c$ & $5150 \mathrm{~m} / \mathrm{s}$ \\
Long-time decoherence times ${ }^{9}$ & $\tau_{0}$ & \\
at $T=0 \mathrm{~K}$ & & $2 \mathrm{~ns}$ \\
at $T=10 \mathrm{~K}$ & & $0.5 \mathrm{~ns}$ \\
\hline
\end{tabular}

TABLE I: System parameters used in the calculations.

\section{PHONON PERTURBATION DURING A QUANTUM GATE}

In the interaction picture with respect to $H_{\mathrm{sc}}$ the system evolution is generated by the Hamiltonian

$$
H=\frac{1}{2} f(t) \sigma_{x}+\frac{1}{2} f(t) \sigma_{y} \hat{R}(t)
$$

where

$$
\hat{R}(t)=i \sum_{\boldsymbol{k}} G(\boldsymbol{k})\left(e^{-i \omega_{\boldsymbol{k}} t} \beta_{-\boldsymbol{k}}-e^{i \omega_{\boldsymbol{k}} t} \beta_{\boldsymbol{k}}^{\dagger}\right)
$$

and $\sigma_{x, y}$ are Pauli matrices.

The evolution in the absence of phonon perturbation is described by the unitary operator $U_{0}(t)$ generated by the first term in Eq. (11),

$$
U_{0}(t)=\cos \frac{\Phi(t)}{2}-i \sin \frac{\Phi(t)}{2} \sigma_{x}, \quad \Phi(t)=\frac{1}{\hbar} \int_{t_{0}}^{t} d \tau f(\tau) .
$$

The effect of the phonon coupling on the driven dynamics may be calculated using the second-order (Born) expansion of the evolution equation for the density matrix ${ }^{16.31}$, including the first term in Eq. (11) exactly and the second one as a perturbation. The reduced density matrix of the qubit is written as

$$
\rho(t)=U_{0}(t)\left[\rho_{0}+\rho^{(2)}(t)\right] U_{0}^{\dagger}(t),
$$

where the correction $\rho^{(2)}(t)$ is calculated from a perturbation expansion

$$
\begin{aligned}
& \rho^{(2)}(t)= \\
& -\frac{1}{\hbar^{2}} \int_{t_{0}}^{t} d \tau \int_{t_{0}}^{t} d \tau^{\prime} \Theta\left(\tau-\tau^{\prime}\right) \operatorname{Tr}_{\mathrm{R}}\left[\tilde{V}(\tau),\left[\tilde{V}\left(\tau^{\prime}\right), \varrho_{0}\right]\right]
\end{aligned}
$$

Here $\operatorname{Tr}_{\mathrm{R}}$ is the trace with respect to the reservoir (phonon) degrees of freedom and $\tilde{V}(t)=U_{0}^{\dagger}(t) V U_{0}(t)$, where $V$ is the second term in Eq. (11). We will assume the initial state of the compound system in the form $\varrho_{0}=\rho_{0} \otimes \rho_{T}$, where $\rho_{T}$ is the thermal equilibrium state of the phonon bath and the initial state of the qubit subsystem is pure, $\rho_{0}=\left|\psi_{0}\right\rangle\left\langle\psi_{0}\right|$.

Defining the operator

$$
Y(\omega)=\frac{1}{\hbar} \int_{t_{0}}^{t} d \tau U_{0}^{\dagger}(\tau) \frac{1}{2} f(\tau) \sigma_{y} U_{0}(\tau) e^{i \omega \tau}
$$


and the phonon spectral function

$$
R(\omega)=\frac{1}{2 \pi} \int d t\langle\hat{R}(t) \hat{R}\rangle e^{i \omega t}
$$

and representing the Heaviside function as

$$
\Theta(t)=-e^{i \omega t} \int \frac{d \omega^{\prime}}{2 \pi i} \frac{e^{-i \omega^{\prime} t}}{\omega^{\prime}-\omega+i 0^{+}},
$$

one may write

$$
\rho^{(2)}(t)=-i\left[h_{t}, \rho_{0}\right]-\frac{1}{2}\left\{A_{t}, \rho_{0}\right\}+\hat{\Phi}_{t}\left[\rho_{0}\right],
$$

with

$$
\begin{aligned}
A_{t} & =\int d \omega R(\omega) Y^{\dagger}(\omega) Y(\omega), \\
\hat{\Phi}_{t}[\rho] & =\int d \omega R(\omega) Y(\omega) \rho Y^{\dagger}(\omega), \\
h_{t} & =\int d \omega R(\omega) \mathcal{P} \int \frac{d \omega^{\prime}}{2 \pi} \frac{Y^{\dagger}\left(\omega^{\prime}\right) Y\left(\omega^{\prime}\right)}{\omega^{\prime}-\omega},
\end{aligned}
$$

where $\mathcal{P}$ denotes the Cauchy principal value.

The explicit form of the operator $Y(\omega)$ is

$$
\left.Y(\omega)=\frac{i}{2} F(\omega)|+\rangle-\left|+\frac{i}{2} F^{*}(-\omega)\right|-\right\rangle+\mid,
$$

where $| \pm\rangle=(|0\rangle \pm|1\rangle) / \sqrt{2}$ and $F(\omega)$ is a nonlinear spectral characteristics of the control field,

$$
F(\omega)=\frac{1}{\hbar} \int_{-\infty}^{\infty} d t e^{i \omega t} f(t) e^{i \Phi(t)} .
$$

We assumed here that the initial time $t_{0}$ is before the control field has been switched on and the final time $t$ is after it has been switched off so that the limits of integration in the above formulas could be extended to $\pm \infty$.

To measure the effect of the phonon-induced dephasing on a quantum gate we will use the error $\delta$, defined as $\delta=1-F^{2}$, where $F=\left\langle\psi_{0}\left|U_{0}^{\dagger}(\infty) \rho(\infty) U_{0}(\infty)\right| \psi_{0}\right\rangle^{1 / 2}$ is the fidelity distance measure ${ }^{32}$ between the ideal (unperturbed) final state $U_{0}(\infty)\left|\psi_{0}\right\rangle\left\langle\psi_{0}\right| U_{0}^{\dagger}(\infty)$ and the actual state $\rho(\infty)$. Substituting Eq. (12) into this definition one finds

$$
\delta=-\left\langle\psi_{0}\left|\rho^{(2)}(\infty)\right| \psi_{0}\right\rangle=\int d \omega R(\omega)\left|\left\langle\psi_{0}^{\perp}|Y(\omega)| \psi_{0}\right\rangle\right|^{2}
$$

where we used the explicit expression (15) and $\left|\psi_{0}^{\perp}\right\rangle$ is a state orthogonal to $\left|\psi_{0}\right\rangle$ in the logical qubit space. Choosing

$$
\begin{aligned}
\psi_{0} & =\sin \frac{\theta}{2}|+\rangle-e^{i \varphi} \cos \frac{\theta}{2}|-\rangle, \\
\psi_{0}^{\perp} & =\cos \frac{\theta}{2}|+\rangle+e^{i \varphi} \sin \frac{\theta}{2}|-\rangle,
\end{aligned}
$$

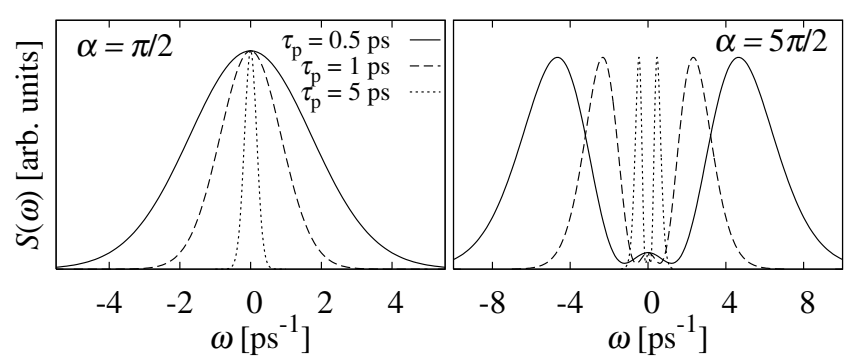

FIG. 1: The spectral characteristics of the driving, $S(\omega)$, for two rotation angles on the qubit Bloch sphere and for a series of pulse durations as shown, for Gaussian pulses [Eq. (10)]. The scales on the two figures are different.

one gets

$$
\begin{aligned}
& \left|\left\langle\psi_{0}^{\perp}|Y(\omega)| \psi_{0}\right\rangle\right|^{2}= \\
& \quad \frac{1}{4}\left|F(\omega) e^{i \varphi} \cos ^{2} \frac{\theta}{2}-F^{*}(-\omega) e^{-i \varphi} \sin ^{2} \frac{\theta}{2}\right|^{2} .
\end{aligned}
$$

Since, in general, the qubit may initially be in any state, we average the error over the Bloch sphere of initial states, i.e., over the angles $(\theta, \varphi)$, which yields

$$
S(\omega)=\left|\left\langle\psi_{0}^{\perp}|Y(\omega)| \psi_{0}\right\rangle\right|_{\mathrm{av}}^{2}=\frac{1}{12}\left(|F(\omega)|^{2}+|F(-\omega)|^{2}\right) .
$$

Since this is even in $\omega$, the error can be written in the form

$$
\delta=\int_{0}^{\infty} d \omega \operatorname{coth}\left(\frac{\hbar \omega}{2 k_{\mathrm{B}} T}\right) \frac{J(\omega)}{\omega^{2}} S(\omega)
$$

where

$$
J(\omega)=\sum_{\boldsymbol{k}}|G(\boldsymbol{k})|^{2} \omega_{\boldsymbol{k}}^{2} \delta\left(\omega-\omega_{\boldsymbol{k}}\right)
$$

is the standard spectral density of the phonon reservoir.

From Eq. (19) it can be seen that $F(\omega)$, and therefore also $S(\omega)$, show simple scaling behavior with respect to the duration of the control pulse. Namely, for a family of pulses of a fixed shape and area, $f(t)=\left(\hbar \alpha / \tau_{0}\right) \tilde{f}\left(t / \tau_{0}\right)$, where $\tilde{f}(x)$ is the fixed shape of the pulse and $\tau_{0}$ is the pulse duration, the spectral function scales as $S(\omega)=$ $\tilde{S}\left(\tau_{0} \omega\right)$ (see Fig. 1). Therefore, for sufficiently long control pulses the error is determined by the low frequency part of the phonon spectral density. To be specific, it can be seen from Eq. (20) that if, for low frequencies, $J(\omega) \sim \omega^{n}$ then $\delta \sim \tau_{0}^{-n+1}$ or $\delta \sim \tau_{0}^{-n+2}$ for long enough pulses at low and high temperatures, respectively ${ }^{17}$. Obviously, slowing down the driving in order to reduce the error is possible only to a certain extent, since for longer durations the error becomes dominated by decoherence effects related, e.g., to finite exciton lifetime and phononassisted transitions, which accumulate with time (linearly for short times). The interplay of these two sources of dephasing creates a trade-off situation and sets a lower 


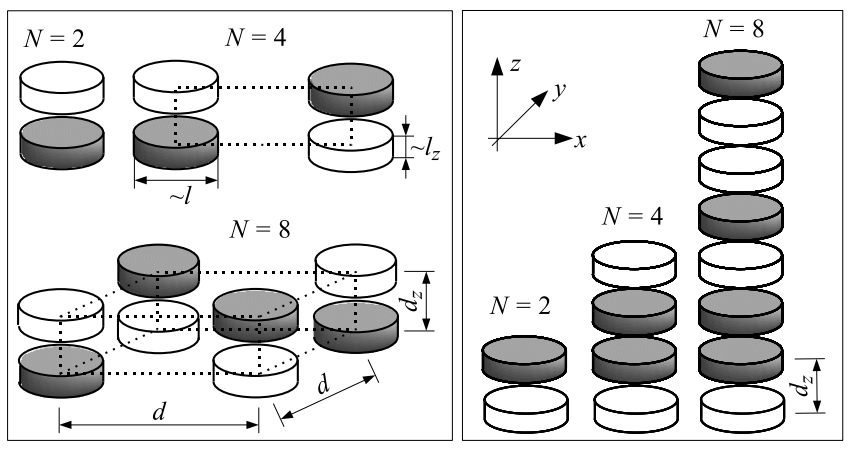

FIG. 2: The two layouts of the QD array: 3-dimensional (left) and linear (right). The dark dots are occupied in the logical $|1\rangle$ state and the white dots in the $|0\rangle$ state. In the linear layout, the logical qubit with $2 N$ dots is constructed by interchanging occupied and empty dots in the $N$ dot qubit and appending it to the original one.

bound on the total error achievable for various pulse durations 17 .

Although, in general, the phonon spectral density for a single exciton confined in a QD depends on the dot geometry, the low frequency behavior is only material-specific (see Appendix) and can only be modified by engineering the properties of phonon modes ${ }^{20.21}$. However, as we show in the next Section, the low-frequency behavior of the effective phonon spectral density may be modified by collective encoding in an array of QDs, which allows one to reduce the total error.

\section{ERROR REDUCTION BY COLLECTIVE ENCODING}

We consider two kinds of arrays of quantum dots. The first one, referred to as 3-dimensional (or 3D), is formed by stacking 2 QDs along the strong confinement $(z)$ axis, arranging 4 QDs in a rectangle in the $x z$ plane or placing 8 QDs in the corners of a cuboid (Fig. 2, left). In the second layout, which we will refer to as linear, $N=2^{n}$ dots are arranged along the $z$ axis, as shown and explained in the right part of Fig. 2

For $N=2^{n}$ quantum dots in the 3D layout one finds the coupling constants $G_{n}$ (for an appropriate choice of origin)

$$
\begin{aligned}
G_{1}(\boldsymbol{k}) & =2 i \sin \frac{k_{z} d_{z}}{2} G_{0}(\boldsymbol{k}), \\
G_{2}(\boldsymbol{k}) & =(2 i)^{2} \sin \frac{k_{z} d_{z}}{2} \sin \frac{k_{x} d}{2} G_{0}(\boldsymbol{k}), \\
G_{3}(\boldsymbol{k}) & =(2 i)^{3} \sin \frac{k_{z} d_{z}}{2} \sin \frac{k_{x} d}{2} \sin \frac{k_{y} d}{2} G_{0}(\boldsymbol{k}),
\end{aligned}
$$

where

$$
G_{0}(\boldsymbol{k})=\sqrt{\frac{1}{2 \hbar \rho_{\mathrm{c}} v c^{3} k}}\left(\sigma_{\mathrm{e}}-\sigma_{\mathrm{h}}\right) \mathcal{F}(\boldsymbol{k})
$$

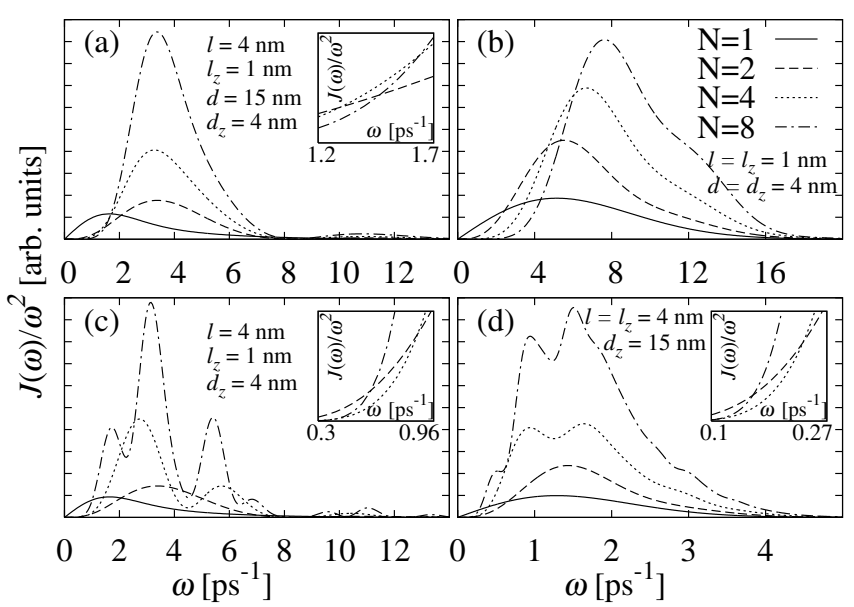

FIG. 3: The phonon spectral densities for the physical excitonic qubit (solid line) and for logical qubits composed of $N$ physical qubits (dashed lines) for two kinds of QD arrays: 3-dimensional $(\mathrm{a}, \mathrm{b})$ and linear $(\mathrm{c}, \mathrm{d})$ with geometrical parameters as indicated.

is the coupling constant for a single dot placed at the origin and the distance parameters $d, d_{z}$ are defined in Fig. 2] From Eq. (21) one then finds the phonon spectral densities for the logical qubits in the low frequency sector,

$$
\begin{aligned}
& J_{1}(\omega) \approx J_{0}(\omega) \frac{d_{z}^{2}}{3 c^{2}} \omega^{2} \approx \frac{\hbar\left(\sigma_{\mathrm{e}}-\sigma_{\mathrm{h}}\right)^{2} d_{z}^{2}}{12 \pi^{2} \rho_{\mathrm{c}} c^{7}} \omega^{5} \\
& J_{2}(\omega) \approx J_{0}(\omega) \frac{d_{z}^{2} d^{2}}{15 c^{4}} \omega^{4} \approx \frac{\hbar\left(\sigma_{\mathrm{e}}-\sigma_{\mathrm{h}}\right)^{2} d_{z}^{2} d^{2}}{60 \pi^{2} \rho_{\mathrm{c}} c^{9}} \omega^{7}, \\
& J_{3}(\omega) \approx J_{0}(\omega) \frac{d_{z}^{2} d^{4}}{105 c^{6}} \omega^{6} \approx \frac{\hbar\left(\sigma_{\mathrm{e}}-\sigma_{\mathrm{h}}\right)^{2} d_{z}^{2} d^{4}}{420 \pi^{2} \rho_{\mathrm{c}} c^{11}} \omega^{9},
\end{aligned}
$$

where

$$
J_{0}(\omega) \approx \frac{\hbar\left(\sigma_{\mathrm{e}}-\sigma_{\mathrm{h}}\right)^{2}}{4 \pi^{2} \rho_{\mathrm{c}} c^{5}} \omega^{3} .
$$

These spectral densities are plotted in Fig. 31 (a,b).

For the linear array of QDs one finds

$$
G_{n}(\boldsymbol{k})=(2 i)^{n} \prod_{j=-1}^{n-2} \sin \left(2^{j} k_{z} d_{z}\right) G_{0}(\boldsymbol{k})
$$

which leads to the spectral density $J_{1}(\omega)$ as above and

$$
\begin{aligned}
& J_{2}(\omega) \approx J_{0}(\omega) \frac{4 d_{z}^{4}}{5 c^{4}} \omega^{4} \approx \frac{\hbar\left(\sigma_{\mathrm{e}}-\sigma_{\mathrm{h}}\right)^{2} d_{z}^{4}}{5 \pi^{2} \rho_{\mathrm{c}} c^{9}} \omega^{7} \\
& J_{3}(\omega) \approx J_{0}(\omega) \frac{64 d_{z}^{6}}{7 c^{6}} \omega^{6} \approx \frac{16 \hbar\left(\sigma_{\mathrm{e}}-\sigma_{\mathrm{h}}\right)^{2} d_{z}^{6}}{7 \pi^{2} \rho_{\mathrm{c}} c^{11}} \omega^{9},(24
\end{aligned}
$$

shown in Fig. 31 c,d).

In both layouts, $J_{n}(\omega) \sim \omega^{2 n+3}$ for small $\omega$ so that for larger logical qubits the spectral density vanishes more quickly for $\omega \rightarrow 0$, which will result in a faster decrease of the pure dephasing error with growing duration of the 

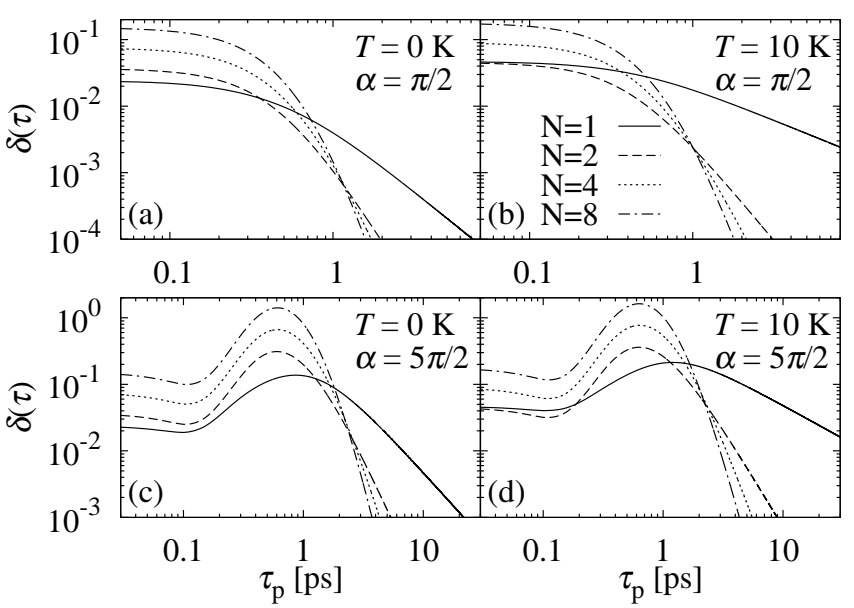

FIG. 4: The phonon-induced dynamical error for a $\pi / 2$ and $5 \pi / 2$ rotation on a physical qubit (solid line) and on a $3 \mathrm{D}$ logical qubit with 2,4 , and 8 dots (dashed and dotted lines) with sizes $l=4 \mathrm{~nm}, l_{z}=1 \mathrm{~nm}$ and separations $d=15 \mathrm{~nm}$, $d_{z}=4 \mathrm{~nm}$, at two different temperatures as shown.

control pulse, as discussed in the previous Section. In order to see this, we calculate the phonon-induced error using Eq. (20) with the appropriate spectral density $J_{n}(\omega)$ [obtained from Eq. (21), for arbitrary $\omega$ ] for various logical qubits.

Let us start the discussion with a logical qubit in the 3D layout, composed of QDs of dimensions typical for self-assembled systems (Fig. 4). First, let us consider the $\pi / 2$ rotation of the qubit (i.e., the single-qubit gate $\left.e^{-i \pi \sigma_{y} / 4}\right)$. For ultrafast pulses $\left(\tau_{\mathrm{p}} \lesssim 100 \mathrm{fs}\right)$, in most cases the error grows with the number of QDs in the array. This regime corresponds to very broad spectral functions $S(\omega)$ (see Fig. 1), so that the whole range of phonon modes contribute to the dephasing. Since considerable destructive interference of phonon excitations is unlikely to appear over the whole wide frequency sector of the phonon spectral density, it may be expected that driving a number of dots simultaneously should lead to more excitation of the phonon reservoir and to more dephasing. In terms of the spectral densities (Fig. 3), this effect is reflected by the growing overall magnitude of $J(\omega)$.

This situation changes for longer pulses $\left(\tau_{\mathrm{p}} \sim 1 \mathrm{ps}\right)$. Now only the long wavelength phonons contribute to dephasing. From the general discussion presented above it is clear that for sufficiently long pulses large collective qubits will lead to lower phonon-induced error than smaller ones due to more favorable scaling of the error with the pulse duration. Indeed, in Fig. 4 the value of $\delta$ decreases for long enough pulses $\left(\tau_{\mathrm{p}} \gtrsim 1 \mathrm{ps}\right)$ according to a power-law dependence with higher negative exponents for larger arrays.

It should be stressed that the reduction of dephasing achieved with collective encoding is not just due to extending the effective system size and the resulting weakening of the overall phonon coupling ${ }^{11.33}$. Such an ef-
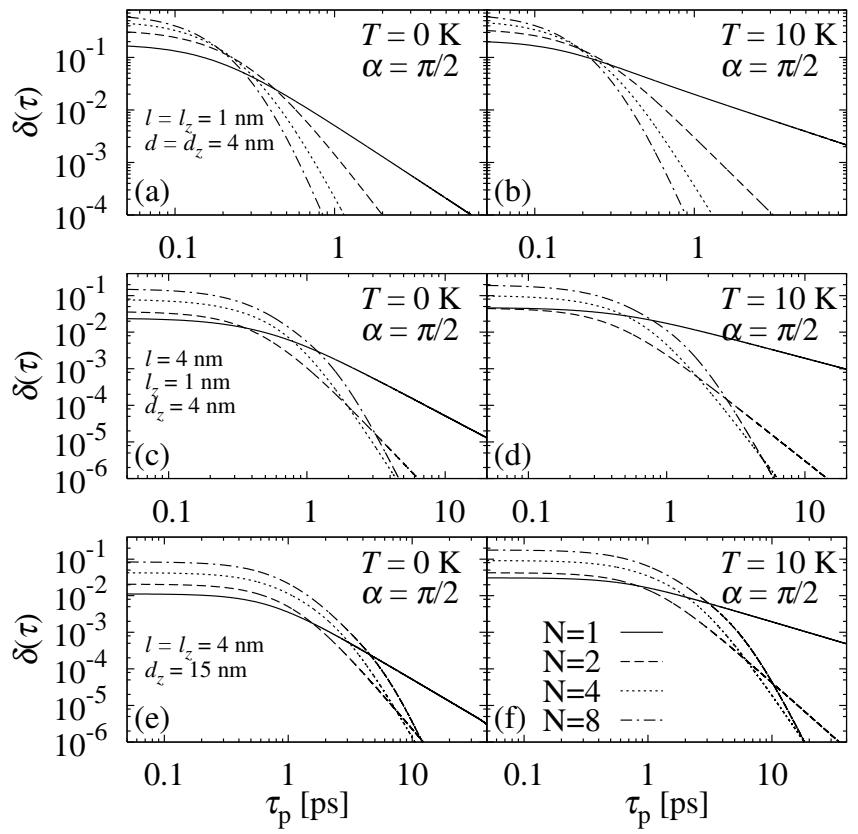

FIG. 5: Comparison of the dynamical error for three different geometries of the QD array: small, closely spaced dots in the 3D layout $(a, b)$, flat dots of moderate size (like in Fig. 4) in the linear layout $(c, d)$ and large dots in the linear layout $(e, f)$.

fect might quantitatively reduce dephasing but could not change the exponent of the low-frequency behavior of $J(\omega)$. Moreover, the reduction of $J(\omega)$ at $\omega \rightarrow 0$ appears only for the special definitions of logical qubits shown in Fig. 2 For a different assignment of physical QDs to the logical qubit states, the dephasing may even be increased, which shows that the effect is actually due to destructive interference between the reservoir excitations caused by the simultaneous switching of the appropriately arranged physical qubits.

For large angles $\alpha$, corresponding to full Rabi rotations around the Bloch sphere, the error dependence on the pulse duration becomes more complicated due to the presence of many maxima in $S(\omega)$ (Fig. 1). While the power-law decrease of $\delta$ at $\tau_{\mathrm{p}} \gtrsim 1$ ps corresponds to the situation when all of $S(\omega)$ lies in the low-frequency sector of $J(\omega)$, the minimum at $\tau_{\mathrm{p}} \sim 100$ fs appears when the area of large $J(\omega)$ coincides with the local minimum between the maxima of $S(\omega)$. Since the collective encoding improves only the low-frequency behavior of $J(\omega)$ only the former is advantageously affected by increasing the size of the QD array.

In the ultrafast limit, the dephasing of a single physical excitonic qubit increases for decreasing dot sizes, since the $1 / l$ momentum cut-off in the formfactor [Eq. (9)] is shifted towards larger momenta and the total area of the phonon spectral density grows. This can also be seen in Fig. 3 (b), corresponding to QDs with the lateral size reduced to $l=1 \mathrm{~nm}$. For longer pulses, this difference in $J(\omega)$ becomes less important since only the low-frequency part of this spectral density is relevant. Indeed, comparing Fig. 5] (a,b) (corresponding to smaller dots) with 
Fig. $4(a, b)$ one notes that the error for ultrashort pulses is much larger for smaller dots but in the region of $\tau_{\mathrm{p}} \gtrsim 1 \mathrm{ps}$ the error for a single dot (solid line) grows very little when the dot size is reduced. On the other hand, smaller dots allow smaller distances $d$ between the physical qubits in the array. As a result, the modulation of $J(\omega)$ resulting from the multi-QD interference attains a longer period $\Delta \omega \sim c / d$ and the sector of low frequencies, where the spectral densities $J_{n}(\omega)$ are ordered according to their asymptotic power-law behavior, extends [see Fig. 3(b)]. Now, the improved behavior of the spectral densities is fully reflected by the reduced values of errors for growing number of QDs in the array already for $\tau_{\mathrm{p}} \gtrsim 300 \mathrm{fs}$.

The linear layout allows the dots to be tightly stacked along the direction of smallest size (growth direction of the semiconductor structure) but, on the other hand, results in a growing distance between the physical qubits on the opposite ends of the chain. This leads to a quickly growing pre-factor at the low-frequency power-law expression [compare Eqs. 22a and (24a)]. As a result, the spectral densities for larger arrays cross $J_{2}(\omega)$ at very low frequencies (see insets in Fig. 3) and one needs longer pulses to take advantage of the lower values of $J_{n}(\omega)$ for $n>1$, as can be seen in Fig. 3 (c,d).

Obviously, the quantitative results presented here depend on the interplay of the physical coupling parameters and the wave number dependence of the original (physical) coupling in the long wave length limit. In any case, however, the spacing between the dots is crucial. This is illustrated by Fig. $5(\mathrm{E}, \mathrm{f})$, where the total error for a linear array of large and widely spaced dots is plotted. From the spectral density shown in Fig. 3(d) it is clear that such a geometry leads not only to the overall narrowing of the phonon spectral characteristics (due to large size) but also to the squeezing of the region when the functions $J(\omega)$ show the advantageous low-frequency behavior. As a result, increasing array size leads to error reduction only for very long pulses.

In a real system, the dynamically induced pure dephasing is not the only error source. Additional error comes from the finite exciton lifetime and other processes, including phonon-assisted transitions to excited exciton states 10.34 , dephasing by phonon scattering 35.36 and probably other effects whose exact nature seems to be poorly understood. The common feature of all these dephasing sources is that they can be described within a Markovian model leading to an exponential decay of coherence with a single time constant $\tau_{0}$ which is experimentally accessible via exciton line broadening. For $\tau_{\mathrm{p}} \ll \tau_{0}$ their contribution to the overall error grows linearly with the gate duration $\tau_{\mathrm{p}}$. Therefore, we add a contribution of $\delta_{0}=\tau_{\mathrm{p}} / \tau_{0}$ to the total error. For brevity, this additional contribution will be referred to as related to "finite lifetime". Depending on the physical process underlying this error, the time constant $\tau_{0}$ may depend in various ways on the number of physical qubits in the array. Here, we will consider two scenarios. In the first one, $\tau_{0}$ is independent of $N$ (which assumes some kind

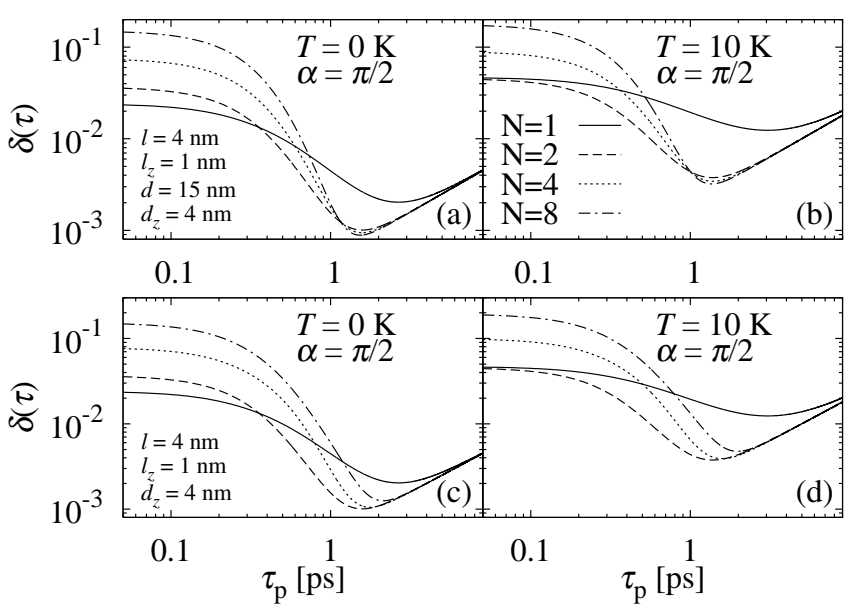

FIG. 6: The total error, including a contribution from finite exciton lifetime and other long-time scale decoherence processes for typical flat dots (with sizes as shown) in 3D (a,b) and linear $(\mathrm{c}, \mathrm{d})$ layout at two different temperatures.

of collective action of the dephasing), while in the second one it is proportional to $1 / N$ (which corresponds to individual decoherence of the physical qubits).

Reaching the low frequency regime, necessary for reducing the dynamical error, requires long control pulses and leads to growing values of $\delta_{0}$. As a result, for long pulse durations the error is always dominated by the linearly growing finite lifetime contribution. The interplay of the dynamical phonon-induced error (growing for short control pulses) and the finite lifetime decoherence (increasing for long pulses) leads to well-defined driving conditions where the error has its minimum ${ }^{17}$. As shown in Fig. [6] in the first model of decoherence (with lifetimes $\tau_{0}=2 \mathrm{~ns}$ and $0.5 \mathrm{~ns}$ at $T=0 \mathrm{~K}$ and $10 \mathrm{~K}$, respectively ${ }^{9}$ ) the optimal total error is reduced by a factor of 2 at $T=0$ $\mathrm{K}$ and almost by a factor of 4 at $T=10 \mathrm{~K}$. It is interesting to note that for these life times large logical qubits $N>2$ bring very little improvement over the simplest one $(N=2)$ and only in the $3 \mathrm{D}$ layout.

For the second decoherence model, the reduction of the effective lifetime of the array considerably restricts the possible error reduction. At $T=0$ one obtains only a fidelity gain of $17 \%$. More error reduction (by a factor of 2) is achievable at higher temperatures. Due to large increase of the error with growing number of physical qubits, only the encoding in $N=2$ physical qubits brings the desired effect.

Thus, nanosecond decoherence times observed in the currently manufactured structures strongly restrict the degree of reduction of dephasing achievable with collective encoding, especially in the independent decoherence model. For realistic sizes and separations of the QDs in the array, taking advantage of the reduced spectral density requires pulses of durations exceeding $1 \mathrm{ps,} \mathrm{which}$ immediately leads to an error of order of $10^{-3}$, further growing for increasing number of dots in the array. However, rapid progress of QD engineering may lead to sys- 


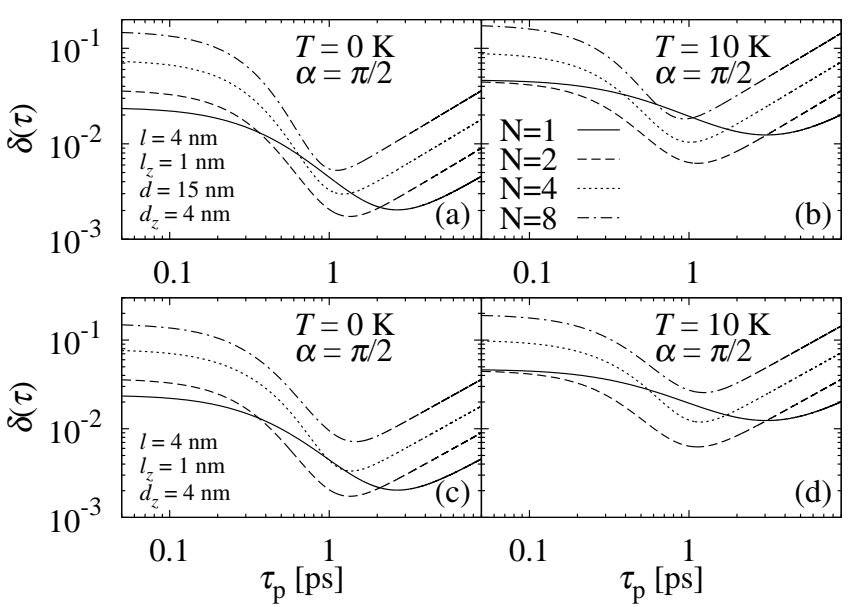

FIG. 7: As in Fig. [6] but with decay probability proportional to the number of physical qubits.

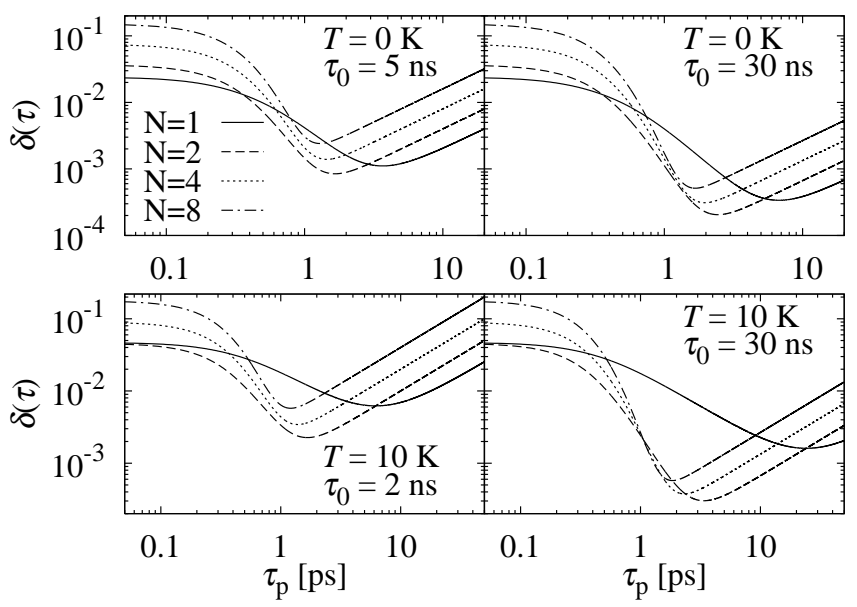

FIG. 8: The total error for a 3D logical qubit with geometry as in Fig. 7 (a,b) for (hypothetical) long characteristic times of the exciton lifetime, with decay-related error proportional to the number of physical qubits, for $\alpha=\pi / 2$ at two temperatures.

tems with considerably longer coherence times, as suggested by the observation of long-lived excitons in particular QD systems ${ }^{37}$. An advantage of the collective qubit is that such reduction of the long time decoherence is much more effectively reflected by the reduction of the total error. As can be seen in Fig. 8 extending the exciton lifetime leads to a reduced error both for a single physical qubit and for a collective qubit. However, the error reduction is much larger for the logical qubit. For instance, the 2-QD encoding (Fig. 17) at $T=0$ reduces the error by $17 \%$ for $\tau_{0}=2 \mathrm{~ns}$, which grows to $31 \%$ if the lifetime is extended to $5 \mathrm{~ns}$ and to a factor of almost 1.7 for $\tau_{0}=30 \mathrm{~ns}$. There is even more gain at higher temperatures. For $T=10 \mathrm{~K}$, one obtains an error reduction by a factor of 2.8 if the lifetime is extended to $2 \mathrm{~ns}$ (which is the actual radiatively limited value in some structures ${ }^{9}$ ) and by almost an order of magnitude for $\tau_{0}=30 \mathrm{~ns}$.

\section{CONCLUSIONS AND OUTLOOK}

The discussion presented above shows that engineering of the effective phonon spectral density by collective encoding of a logical qubit in an array of physical (excitonic) qubits may lead to a reduction of the phonon-induced dephasing during control operations on the qubit. This is possible since for long enough pulses (slow driving) only long-wavelength part of the phonon reservoir is involved in the dephasing dynamics which extends the effective correlation length of the reservoir and opens the possibility for collective encoding. Such collective encoding reduces the total error resulting from the joint action of various dephasing mechanisms and increases the gain from possible further extension of the exciton life times. With the collective encoding it seems feasible to reduce the total error for a single-qubit operation to values of order of $10^{-3}$ even at the relatively high temperature of $T=10 \mathrm{~K}$, which is remarkable in view of the recent progress in the quantum error correction theory under realistically strong dephasing 38 .

The present analysis was restricted to Gaussian pulses. It is known, however, that pulse shaping 19 or pulsed control techniques 18 lead to reduced phonon dephasing effect. The reach structure of the effective phonon spectral density for collective qubits suggests that combining the pulse shaping techniques with the collective encoding may be very fruitful. Another possibility of reducing the overall error might be to combine the collective encoding against dynamical dephasing with the noiseless (subradiant) encoding against radiative decay ${ }^{22}$.

In more general terms, our discussion shows that certain details of the phonon spectral density may be of essential importance for the dephasing of a system driven by finite pulses. Obviously, the system response to an ultrashort pulse, as observed in optical experiments 10 , yields valuable information on the overall strength of the phonon-induced dephasing. However, we have seen that the degree of decoherence for finite pulses is governed only by the low-frequency part of the relevant spectral function and may decrease even though the dephasing in the ultrafast limit increases (e.g., for arrays of smaller but more closely spaced QDs). It is therefore possible that systems based on various materials ${ }^{33}$ and restricted geometries ${ }^{20}$ may offer previously unnoticed possibilities in the slow driving regime. Understanding of the peculiarities of the carrier-phonon dynamics underlying the dephasing of driven systems may also be helpful for designing robust schemes for the optical control of spin qubits.

Supported by the Polish Ministry of Scientific Research and Information Technology (PBZ-MIN-008/P03/2003). P.M. is grateful to A. von Humboldt Foundation for support. 


\section{APPENDIX A: UNIVERSALITY OF CARRIER-PHONON COUPLING IN THE LONG WAVE LENGTH LIMIT}

In this Appendix we show that for small variation of individual dot sizes and for small difference between the electron and hole localization in a dot, the excitonphonon coupling constant can be written in the form (2), with the formfactor replaced by Eq. (9) which depends only on the average width of the electron and hole wave functions in the QD array.

In general, the electron and hole wave functions in the $n$th dot may be characterized by different localization widths $l_{n}^{(\mathrm{e} / \mathrm{h})}$ (for simplicity, we still assume that the exciton wave function is a product of Gaussians isotropic in the $x y$ plane and with a fixed width $l_{z}$ ). The carrierphonon interaction is then described by Eq. (2) with 27

$$
\mathcal{F}_{n}(\boldsymbol{k})=\widetilde{\sigma}_{\mathrm{e}} e^{-k_{\perp}^{2} l_{n}^{(\mathrm{e}) 2} / 4-k_{z}^{2} l_{z}^{2} / 4}-\widetilde{\sigma}_{\mathrm{h}} e^{-k_{\perp}^{2} l_{n}^{(\mathrm{h}) 2} / 4-k_{z}^{2} l_{z}^{2} / 4},
$$

where $\widetilde{\sigma}_{\mathrm{e} / \mathrm{h}}=\sigma_{\mathrm{e} / \mathrm{h}} /\left(\sigma_{\mathrm{e}}-\sigma_{\mathrm{h}}\right)$. We define the averaged wave function width

$$
l^{2}=\frac{1}{2 N}\left[\sum_{n}\left(l_{n}^{(\mathrm{e})}\right)^{2}+\sum_{n}\left(l_{n}^{(\mathrm{h})}\right)^{2}\right]
$$

and the deviations from the average

$$
\left(\Delta l_{n}^{(\mathrm{e} / \mathrm{h})}\right)^{2}=\left(l_{n}^{(\mathrm{e} / \mathrm{h})}\right)^{2}-l^{2}
$$

The formfactor can now be written as

$$
\begin{aligned}
\mathcal{F}_{n}(\boldsymbol{k})= & e^{-k_{\perp}^{2} l^{2} / 4-k_{z}^{2} l_{z}^{2} / 4} \\
& \times\left[\widetilde{\sigma}_{\mathrm{e}} e^{-k_{\perp}^{2}\left(\Delta l_{n}^{(\mathrm{e})}\right)^{2} / 4}-\widetilde{\sigma}_{\mathrm{h}} e^{-k_{\perp}^{2}\left(\Delta l_{n}^{(\mathrm{h})}\right)^{2} / 4}\right] .
\end{aligned}
$$

It is clear that $k_{\perp}$ is cut off at the value of $\sim 1 / l$, hence $k_{\perp} \Delta l_{n}^{(\mathrm{e} / \mathrm{h})} \ll 1$ if $\Delta l_{n}^{(\mathrm{e} / \mathrm{h})} \ll l$, i.e., when the variation of dot sizes is relatively small. The exponents may be therefore expanded into power series,

$$
\begin{aligned}
\mathcal{F}_{n}(\boldsymbol{k})= & e^{-k_{\perp}^{2} l^{2} / 4-k_{z}^{2} l_{z}^{2} / 4} \\
& \times\left[1-\widetilde{\sigma}_{\mathrm{e}} \frac{k_{\perp}^{2}\left(\Delta l_{n}^{(\mathrm{e})}\right)^{2}}{4}-\widetilde{\sigma}_{\mathrm{h}} \frac{k_{\perp}^{2}\left(\Delta l_{n}^{(\mathrm{h})}\right)^{2}}{4}\right],
\end{aligned}
$$

where we took into account that $\widetilde{\sigma}_{\mathrm{e}}-\widetilde{\sigma}_{\mathrm{h}}=1$. Retaining only the leading term one arrives at Eq. (9). It should be noted that independently of the shape of the wave functions, normalization condition requires that for long wavelengths $\mathcal{F}_{n}(\boldsymbol{k})=1+O\left(k^{2}\right)$, which is sufficient for deriving Eqs. (22a,c) and (24a b).
* Electronic address: Anna.Grodecka@pwr.wroc.pl

1 P. Zanardi and F. Rossi, Phys. Rev. Lett. 81, 4752 (1998).

${ }^{2}$ E. Biolatti, R. C. Iotti, P. Zanardi, and F. Rossi, Phys. Rev. Lett. 85, 5647 (2000).

3 X. Li, Y. Wu, D. Steel, D. Gammon, T. Stievater, D. Katzer, D. Park, C. Piermarocchi, and L. Sham, Science 301, 809 (2003).

4 E. Pazy, E. Biolatti, T. Calarco, I. D'Amico, P. Zanardi, F. Rossi, and P. Zoller, Europhys. Lett. 62, 175 (2003).

5 T. Calarco, A. Datta, P. Fedichev, E. Pazy, and P. Zoller, Phys. Rev. A 68, 12310 (2003).

${ }^{6}$ Pochung Chen, C. Piermarocchi, L. J. Sham, D. Gammon, and D. G. Steel, Phys. Rev. B 69, 075320 (2004).

7 F. Troiani, E. Molinari, and U. Hohenester, Phys. Rev. Lett. 90, 206802 (2003).

8 A. Zrenner, E. Beham, S. Stufler, F. Findeis, M. Bichler, and G. Abstreiter, Nature 418, 612 (2002).

${ }^{9}$ W. Langbein, P. Borri, U. Woggon, V. Stavarache, D. Reuter, and A. D. Wieck, Phys. Rev. B 70, 033301 (2004).

10 P. Borri, W. Langbein, S. Schneider, U. Woggon, R. L. Sellin, D. Ouyang, and D. Bimberg, Phys. Rev. Lett. 87, 157401 (2001).

11 B. Krummheuer, V. M. Axt, and T. Kuhn, Phys. Rev. B 65, 195313 (2002).

12 A. Vagov, V. M. Axt, and T. Kuhn, Phys. Rev. B 67, 115338 (2003).

13 A. Vagov, V. M. Axt, and T. Kuhn, Phys. Rev. B 66,
165312 (2002).

14 L. Jacak, P. Machnikowski, J. Krasnyj, and P. Zoller, Eur. Phys. J. D 22, 319 (2003).

15 K. Roszak and P. Machnikowski, Phys. Lett. A, in press (2006), cond-mat/0504135

16 K. Roszak, A. Grodecka, P. Machnikowski, and T. Kuhn, Phys. Rev. B 71, 195333 (2005).

17 R. Alicki, M. Horodecki, P. Horodecki, R. Horodecki, L. Jacak, and P. Machnikowski, Phys. Rev. A 70, 010501(R) (2004).

18 V. M. Axt, P. Machnikowski, and T. Kuhn, Phys. Rev. B 71, 155305 (2005).

19 U. Hohenester and G. Stadler, Phys. Rev. Lett. 92, 196801 (2004).

20 B. Krummheuer, V. M. Axt, and T. Kuhn, Phys. Rev. B 72, 245336 (2005).

21 M. Cazayous, J. Groenen, J. R. Huntzinger, G. Bachelier, A. Zwick, A. Mlayah, E. Bedel-Pereira, F. Negri, H. Carrère, N. Bertru, C. Paranthoen, and O. Dehaese, Phys. Rev. B 69, 125323 (2004).

22 P. Zanardi and M. Rasetti, Phys. Rev. Lett. 79, 3306 (1997).

23 D. Bacon, J. Kempe, D. A. Lidar, and K. B. Whaley, Phys. Rev. Lett. 85, 1758 (2000).

24 D. A. Lidar, D. Bacon, J. Kempe, and K. B. Whaley, Phys. Rev. A 63, 022306 (2001).

25 D. A. Lidar, D. Bacon, J. Kempe, and K. B. Whaley, Phys. 
Rev. A 63, 022307 (2001).

${ }^{26}$ P. Zanardi and F. Rossi, Phys. Rev. B 59, 8170 (1999).

27 A. Grodecka, L. Jacak, P. Machnikowski, and K. Roszak, in Quantum Dots: Research Developments, edited by P. A. Ling (Nova Science Publishers, NY, 2005), p. 47.

${ }^{28}$ G. D. Mahan, Many-Particle Physics (Kluwer, New York, 2000).

29 B. W. Lovett, J. H. Reina, A. Nazir, B. Kothari, and G. A. D. Briggs, Phys. Lett. A 315, 136 (2003).

30 B. W. Lovett, J. H. Reina, A. Nazir, and G. A. D. Briggs, Phys. Rev. B 68, 205319 (2003).

31 R. Alicki, M. Horodecki, P. Horodecki, and R. Horodecki, Phys. Rev. A 65, 062101 (2002).

32 M. A. Nielsen and I. L. Chuang, Quantum Computation and Quantum Information (Cambridge University Press, Cambridge, 2000).

33 B. Krummheuer, V. M. Axt, T. Kuhn, I. D'Amico, and F. Rossi, Phys. Rev. B 71, 235329 (2005).

34 P. Borri, W. Langbein, S. Schneider, U. Woggon, R. L. Sellin, D. Ouyang, and D. Bimberg, Phys. Stat. Sol. (b) 233, 391 (2002).

35 E. A. Muljarov and R. Zimmermann, Phys. Rev. Lett. 93, 237401 (2004).

36 P. Machnikowski, quant-ph/0504035

37 T. Lundstrom, W. Shoenfeld, H. Lee, and P. M. Petroff, Science 286, 2312 (1999).

38 E. Knill, Nature 434, 39 (2005). 